\title{
Uso do estimulante de colônia de granulócitos nas neutropenias em cães e gatos
}

\author{
Granulocyte colony-stimulating factor use in neutropenias in dogs and cats
}

\author{
Cynthia de Assumpção Lucidi ${ }^{\mathrm{I}}$ Regina Kiomi Takahira ${ }^{\mathrm{II}}$
}

\section{- REVISÃO BIBLIOGRÁFICA -}

\section{RESUMO}

As neutropenias persistentes podem ser decorrentes de alterações na granulopoiese, causadas por efeitos supressivos ou tóxicos à medula óssea, predispõem o paciente a infecções comprometendo sua sobrevida. As neutropenias intensas decorrentes de toxicidade por quimioterápicos podem requerer a suspensão temporária ou permanente do medicamento, podendo gerar resistência das células neoplásicas ao tratamento. $O$ uso de fatores de crescimento hematopoiético recombinantes em animais tem aumentado muito nos últimos anos, devido a sua crescente disponibilidade na medicina humana. O fator estimulante de colônia para granulócitos recombinante humano (rhG-CSF) age aumentando o número de neutrófilos circulantes e possui grande potencial para amenizar ou reverter quadros de neutropenia associada a condições de mielotoxicidade e mielosupressão em cães e gatos.

Palavras-chave: G-CSF, neutrófilos, granulopoiese, neutropenia.

\section{ABSTRACT}

Persistent neutropenias can occur after granulopoiesis disturbances caused by myelosupressive or myelotoxic effects, and predispose patients to infections and impairs their survival. Furthermore, severe chemotherapyinduced neutropenias must require temporary or definitive treatment interruption, what may lead to drug-resistance of neoplastic cells. The use of recombinant stem cell factors in animals has been increasing due to its bigger disponibility for human beings. The human recombinant granulocyte colonystimulating factor (rhG-CSF) increases neutrophil numbers in peripheral blood and has great potential to alleviate or revert myelotoxic or myelosupression neutropenias in dogs and cats.

Key words: G-CSF, neutrophils, granulopoiesis, neutropenia.

\section{INTRODUÇÃO}

Efeitos mielotóxicos e mielosupressivos podem afetar a granulopoiese e resultar em neutropenias persistentes, que predispõem o paciente a infecções e comprometem sua qualidade de vida e sobrevida (CHUN et al., 2001; COUTO, 2003). O fator estimulante de colônia para granulócitos recombinante humano (rhG-CSF) tem como principal efeito o aumento do número de neutrófilos circulantes e possui grande potencial para reduzir a duração e intensidade da neutropenia associada às condições de mielotoxicidade e mielosupressão (OBRADOVICH et al., 1991; MISHU et al., 1992; HENRY et al., 1998; FELDMAN et al., 2000). Pretende-se, com este trabalho, discutir o uso do rhGCSF em cães e gatos, a partir da compreensão da fisiologia da granulopoiese e das principais causas de neutropenia.

Fisiologia dos granulócitos

O conhecimento da fisiologia da granulopoiese é fundamental para a compreensão dos mecanismos de ação e indicações do uso do rhG-CSF em cães e gatos. Nestes animais, os leucócitos são produzidos na medula óssea e dividem-se em granulócitos (neutrófilos, eosinófilos e basófilos) e mononucleares (monócitos e linfócitos). Todos eles se originam da mesma célula tronco pluripotencial, que

IFaculdade de Medicina Veterinária e Zootecnia (FMVZ), Universidade Estadual Paulista (UNESP), Departamento de Clínica Veterinária (DCV), 18618-000, Botucatu, SP, Brasil. Distrito de Rubião Jr., s/nº. E-mail: cylucidi@yahoo.com.br. Autor para correspondência.

"FMVZ, UNESP, DCV, Botucatu, SP, Brasil. 
tem capacidade de auto-replicação e se diferencia nas unidades formadoras de colônia específicas, dependendo do estímulo a que for submetida (JAIN, 1993).

Três compartimentos ou pools de neutrófilos são reconhecidos na medula óssea: (1) mitótico ou de proliferação, constituído por mieloblastos, promielócitos e mielócitos; (2) de maturação, constituído por metamielócitos e bastonetes; e (3) de reserva, formado por neutrófilos maduros e alguns bastonetes. O tempo de maturação para um mieloblasto se diferenciar e ser liberado como neutrófilo segmentado maduro no sangue periférico é de aproximadamente cinco a seis dias em cães e gatos (STOCKHAM \& SCOTT, 2002). Os neutrófilos diminuem de tamanho, perdem a basofilia citoplasmática e sofrem condensação e segmentação nuclear durante sua maturação. Na medula óssea normal, existe um grande número de células maduras, um número menor de células em maturação e um pequeno número de células imaturas (HARVEY, 2001).

As principais citocinas indutoras da granulopoiese são o fator estimulante de colônia de granulócitos (G-CSF), fator estimulante de colônia de granulócitos e monócitos (GM-CSF) e algumas interleucinas (IL-1, IL-3, IL-5 e IL-6), que aumentam o número de mitoses, diminuem o tempo de maturação e aumentam a liberação de granulócitos para o sangue periférico (FELDMAN et al., 2000; STOCKHAM \& SCOTT, 2002).

O G-CSF é linhagem-específico, ou seja, exerce efeito somente sobre a linhagem neutrofílica (FELDMAN et al., 2000), e é produzido pelas células do estroma da medula óssea, fibroblastos, células endoteliais, monócitos e macrófagos, sob estímulo de citocinas inflamatórias, como IL-1, IL-3 e fator de necrose tumoral (TNF), e endotoxinas bacterianas (HENRY et al., 1998). O fator estimulante de colônia de granulócitos desenvolve importante papel na granulopoiese e atua na medula óssea aumentando a liberação de neutrófilos maduros do compartimento de reserva para a circulação, a proliferação e maturação de precursores granulocíticos e a função dos neutrófilos, aumentando sua capacidade de fagocitose, quimiotaxia e degranulação (HENRY et al., 1998; REWERTS \& HENRY, 1998; FELDMAN et al., 2000).

Os neutrófilos constituem a primeira linha de defesa do organismo, participando de inflamações, infecções, principalmente bacterianas, e lesões teciduais. Estas células possuem grânulos com enzimas proteolíticas, que participam no processo de digestão de antígenos. A cinética dos neutrófilos dentro dos vasos se constitui basicamente de rolamento pelo tecido endotelial, adesão à célula endotelial do local envolvido na quimiotaxia, migração para o tecido lesado e fagocitose de substâncias estranhas ao organismo (STOCKHAM \& SCOTT, 2002).

De acordo com o tempo de vida média dos componentes sanguíneos, é fácil entender que a linhagem granulocítica é a primeira a ser afetada em casos de deficiência na hematopoiese, pois os neutrófilos duram de quatro a oito horas na circulação, ao passo que as plaquetas têm uma vida média de quatro a seis dias, e as hemácias de 60 dias no gato e de 120 dias no cão (CHUN et al., 2001; COUTO, 2003).

\section{Causas de neutropenia}

É importante a compreensão dos mecanismos de neutropenia para o entendimento das indicações e contra-indicações do uso do G-CSF como terapia para aumentar o número de neutrófilos sanguíneos. A redução do número de neutrófilos circulantes pode ser transitória ou persistente e estar ou não relacionada aos problemas de produção pela medula óssea, como será descrito a seguir.

Neutropenia inflamatória: ocorre quando a migração de neutrófilos para os tecidos for maior do que sua liberação pela medula óssea. Está associada principalmente a processos bacterianos graves como sepse e endotoxemia (BROWN \& ROGERS, 2001). Esta resposta é aguda e geralmente a neutropenia se resolve em cinco a sete dias, que é o tempo necessário para a produção e liberação de novos neutrófilos para a circulação (JAIN, 1993).

Neutropenia inflamatória por endotoxemia: endotoxinas de bactérias Gram-negativas levam à liberação de mediadores inflamatórios, que causam a ativação e adesão de neutrófilos ao endotélio vascular, e neutropenia que dura de uma a três horas. A neutropenia tende a ser transitória, pois as endotoxinas estimulam a liberação de neutrófilos da medula óssea em oito a 12 horas e o aumento de sua produção em três a cinco dias (WAGNER \& ROTH, 1999).

Destruição periférica: neutropenia por destruição periférica ocorre quando a destruição de neutrófilos imunomediada ou pelo sistema monocítico fagocitário (SMF) é maior do que a capacidade de produção pela medula óssea (STOCKHAM \& SCOTT, 2002). A neutropenia por destruição imunomediada ainda não está bem estabelecida em pequenos animais, mas ocorre pela presença de anticorpos anti-neutrófilos e geralmente é idiopática ou está associada ao uso de alguns fármacos, infecções, neoplasias, ou outras doenças auto-imunes, como lupus eritematoso sistêmico ou artrite reumatóide (BROWN \& ROGERS, 2001). Contudo, a hiperplasia do SMF está relaciona às 
síndromes hemofagocíticas e pode estar relacionada a outras citopenias (WALTON et al., 1996; WEISS, 2001).

Hipoplasia granulocítica: a hipoplasia da série granulocítica geralmente está associada à hipoplasia concomitante das outras linhagens celulares e representa a principal indicação para o uso dos estimulantes da hematopoiese. Neste caso, a produção de neutrófilos está diminuída devido à destruição das células precursoras ou do microambiente hematopoiético (STOCKHAM \& SCOTT, 2002). A neutropenia é persistente e observa-se hipoplasia granulocítica ao exame da medula óssea (HARVEY, 2001).

Alguns fatores relacionados à hipoplasia da linhagem granulocítica em pequenos animais são erliquiose crônica (AROCH \& HARRUS, 2001), radiação (MAC VITTIE et al., 2005), uso de fármacos como quimioterápicos (COUTO, 1990; KIM \& DEMETRI, 1996; COUTO, 2003), estrógenos (SUTTORP et al., 2002), griseofulvina (HELTON et al., 1986; KUNKLE \& MEYER, 1987), captopril (HOLLAND et al., 1996) e necrose da medula óssea associada à sepse, endotoxemia, neoplasia, coagulação intravascular disseminada, parvovirose, lupus eritematoso sistêmico e hipóxia(HARVEY, 2001).

Produção ineficiente: a produção ineficiente de neutrófilos está associada ao defeito na maturação dos precursores granulocíticos ou à presença de precursores degenerados, que sofrem morte celular antes de saírem da medula óssea. Esta condição geralmente é caracterizada por neutropenia no sangue periférico, apesar da hiperplasia granulocítica na medula óssea (HARVEY, 2001; STOCKHAM \& SCOTT, 2002), e tem sido associada às síndromes mielodisplásicas (WEISS \& AIRD, 2001) e à infecção pelo vírus da imunodeficiência felina (FIV) ou pelo vírus da leucemia felina (FeLV)(LESTER \& SEARCY, 1981; SHELTON et al., 1990).

Hematopoiese cíclica: a neutropenia relacionada à hematopoiese cíclica está associada a um defeito na célula tronco pluripotencial, que ocorre como uma doença hereditária autossômica recessiva presente apenas em cães cinza da raça Collie. Estes animais apresentam oscilações na produção dos fatores estimulantes hematopoiéticos (eritropoietina, trombopoietina, G-CSF, IL-1, IL-6) e conseqüente produção cíclica das células sanguíneas. Estes animais podem apresentar trombocitopenia e anemia discretas, e sofrem altos riscos de infecções e sepse devido à neutropenia, que pode ser intensa (HAMMOND et al., 1990; FELDMAN et al., 2000). Esta doença causa ainda lesões articulares e sistêmicas e tem sido usada como modelo de estudo para a hematopoiese cíclica humana (FELDMAN et al., 2000).
Fator estimulante de colônia de granulócitos

O uso dos fatores de crescimento hematopoiético recombinantes tem crescido muito nos últimos anos, na medicina veterinária e humana, devido ao aumento de sua disponibilidade no mercado. Estas citocinas sintéticas regulam a produção, o desenvolvimento e a função das linhagens hematopoiéticas e são indicadas para reduzir a morbidade relacionada aos efeitos mielosupressivos induzidos por algumas substâncias tóxicas à medula óssea, incluindo quimioterápicos, radiação e infecções virais (FELDMAN et al., 2000).

$O$ fator estimulante de colônia para granulócitos recombinante (rG-CSF) é um dos fatores de crescimento hematopoiético com maior potencial para uso clínico, pois é linhagem-específico e age em precursores granulocíticos, aumentando o número e a função dos neutrófilos (MISHU et al., 1992; HENRY et al., 1998). Além disso, também está associado à supressão da apoptose de neutrófilos procedentes de cães sadios in vitro, levando ao aumento da vida média destas células (OGUMA et al., 2005).

$\mathrm{Na}$ prática, o que se observa em cães é o aumento da liberação de neutrófilos para o sangue uma a duas horas após sua administração e a aceleração da produção em um a três dias (HAMMOND et al., 1991), com o retorno à contagem inicial normal três a cinco dias após a suspensão do medicamento (OBRADOVICH et al., 1991). A resposta ao tratamento depende da sua duração e do número de células tronco viáveis na medula óssea (FELDMAN et al., 2000).

$\mathrm{O}$ rG-CSF foi primeiramente estudado na medicina humana, na qual é indicado no tratamento de neutropenia cíclica, na recuperação após transplante de medula óssea, no aumento do número de células tronco circulantes em doadores de medula óssea, após quimioterapia, nas neutropenias idiopáticas e mielodisplasias, e em pacientes com neutropenia decorrente de síndrome da imunodeficiência adquirida (AIDS) (HENRY et al., 1998).

O uso do rG-CSF foi referido pela primeira vez na medicina veterinária em 1988, no tratamento da neutropenia cíclica do Collie cinza (LOTHROP et al., 1988), e, desde então, tem sido mais estudado, conquistando grande importância no tratamento e na prevenção das neutropenias nos animais de companhia. Este medicamento é indicado apenas nos casos de neutropenias persistentes, nas quais há um defeito na produção ou maturação dos neutrófilos, como nos casos de hipoplasia, hematopoiese cíclica e alguns casos de produção ineficiente, sendo contra-indicado nos casos de neutropenias agudas e transitórias. Desta forma, a realização de hemogramas seriados e do exame 
da medula óssea do paciente são de fundamental importância para a confirmação da necessidade de seu uso.

O G-CSF recombinante canino (rcG-CSF) foi usado com sucesso para aumentar o número de neutrófilos em cães e em gatos, causando neutrofilia poucas horas após sua administração por via subcutânea (OBRADOVICH et al., 1991; MISHU et al., 1992; OBRADOVICH et al., 1993). Entretanto, seu uso é restrito às universidades e centros de pesquisa, fazendo com que o G-CSF recombinante humano (rhGCSF), de nome genérico filgrastima, seja ainda o mais comumente utilizado na prática médica de pequenos animais (FELDMAN et al., 2000). A dose recomendada nestes animais é de $5 \mu \mathrm{g} \mathrm{kg}^{-1}$ dia $^{-1}$, por via subcutânea (HENRY et al., 1998).

$\mathrm{Na}$ medicina veterinária, o rhG-CSF é indicado no tratamento de neutropenias após quimioterapia (BRAVO et al., 1996; COUTO, 1990; HENRY et al., 1998; COUTO, 2003), após radiação (NOTHDURFT et al., 1997; MAC VITTIE et al., 2005), na recuperação após transplante de medula óssea (STORB et al., 1994), na mobilização de células tronco hematopoiéticas para o sangue periférico (THOMASSON et al., 2003), nas neutropenias causadas por fármacos (HOLLAND et al., 1996) e na neutropenia cíclica do Collie cinza (HAMMOND et al., 1990). Seu uso foi sugerido no tratamento de gatos com neutropenias associadas a FIV e FeLV, porém ainda está sob estudos (SHELTON \& LINENBERGER, 1995; HENRY et al., 1998). PHILLIPS et al. (2005) demonstraram que o tratamento com o fator estimulante de colônia de granulócitos recombinante humano em gatos infectados pelo vírus da FIV aumentou o número de neutrófilos circulantes destes animais.

Atualmente a principal aplicação do rhG-CSF na medicina veterinária é na redução da duração e intensidade das neutropenias associadas à quimioterapia em altas doses, já que as neutropenias intensas decorrentes de quimioterapias requerem a suspensão temporária ou permanente do quimioterápico, podendo gerar resistência das células neoplásicas ao tratamento e comprometer a sobrevida do paciente (COUTO, 2003). Nestes pacientes, a terapia com G-CSF é indicada em casos de neutropenia acompanhada de febre $\left(<1.000\right.$ neutrófilos $\left.\mu \mathrm{L}^{-1}\right)$, neutropenia grave e persistente $\left(<500\right.$ neutrófilos $\mu \mathrm{L}^{-1}$, por mais de 72 horas) ou em animais com histórico de neutropenia acompanhada de febre em quimioterapias anteriores (HENRY et al., 1998).

O tratamento com rhG-CSF em pequenos animais é contra-indicado nos casos de sepse, pois não se observou aumento significativo no número de neutrófilos circulantes ou na sobrevida de animais que receberam o medicamento em relação aos animais que não receberam (MISCHKE et al., 2001; QUEZADO et al., 2001). Efeitos benéficos deste medicamento foram observados somente quando sua administração se iniciou dias antes da ocorrência de sepse experimental, aumentando o número de neutrófilos circulantes e aumentando as defesas e a taxa de sobrevivência dos cães estudados (EICHACKER, 1994).

Ainda é controversa a administração de rhGCSF aos animais com hipoplasia granulocítica causada por erliquiose crônica ou intoxicação por estrógenos, devido à baixa viabilidade das células precursoras na medula óssea, sendo indicada por alguns autores (AROCH \& HARRUS, 2001; SUTTORP et al., 2002) e pouco aceita por outros (FELDMAN et al., 2000).

Os fatores de crescimento hematopoiético espécie-específicos raramente induzem o desenvolvimento de anticorpos, porém o uso de rhGCSF, uma proteína recombinante humana, por semanas consecutivas em cães e gatos, pode induzir a formação de anticorpos contra o medicamento, neutralizando sua ação e fazendo com que os neutrófilos voltem aos valores de normalidade (HAMMOND et al., 1991; PHILLIPS et al., 2005). Tratamentos mais prolongados podem induzir a formação de anticorpos contra o GCSF nativo do animal, por meio de reação cruzada. Cães normais recebendo $10 \mathrm{mg} \mathrm{kg}^{-1} \mathrm{dia}^{-1}$, por via subcutânea, durante 30 dias, desenvolveram anticorpos anti-G-CSF canino, resultando em neutropenia persistente por quatro meses após o término da terapia (HAMMOND et al., 1991). O mesmo aconteceu com gatos portadores de FIV, que apresentaram a produção de anticorpos neutralizantes cruzados contra o G-CSF felino endógeno após o uso do rhG-CSF em ciclos de tratamento repetidos e prolongados (PHILLIPS et al., 2005).

Por outro lado, o uso destes fatores recombinantes humanos por poucos dias em cães e gatos não resulta na formação de anticorpos (HENRY et al., 1998; PHILLIPS et al., 2005). O uso repetido por curtos períodos de rhG-CSF em cães portadores de neoplasia não levou à formação de anticorpos contra esta citocina após quatro ciclos de quimioterapia, demonstrando que o seu uso do rhG-CSF pode ser uma opção segura para o tratamento ou a prevenção de neutropenia (HENRY et al., 1998).

No Brasil, o rhG-CSF é produzido por diversos laboratórios e comercializado principalmente por farmácias especializadas em produtos oncológicos. O medicamento se apresenta na forma de ampolas de $1 \mathrm{~mL}$, contendo $300 \mu \mathrm{g}$ de filgrastima, o suficiente para um ciclo de tratamento de cinco a seis dias em um cão 
de $10 \mathrm{~kg}$. Apesar desta ampla disponibilidade, existem poucos estudos nacionais sobre o uso de estimulantes hematopoiéticos em cães e gatos, como um estudo realizado por PEREZ et al. (2005), que avaliou os possíveis efeitos mieloprotetores do decanoato de nandrolona (Deca-durabolin ${ }^{\circledR}$ ), um andrógeno sintético com ação anabolizante, em ratos com depressão medular induzida pelo quimioterápico vincristina $\left(\right.$ Oncovin $\left.{ }^{\circledR}\right)$.

\section{CONCLUSÕES}

O fator estimulante de colônia para granulócitos recombinante humano (rhG-CSF) age aumentando o número de neutrófilos circulantes e possui grande potencial para amenizar ou reverter quadros de neutropenia associada às condições de mielotoxicidade e mielosupressão em cães e gatos.

A ausência de estudos com o rhG-CSF disponível no Brasil limita seu uso como excelente fármaco para o tratamento das neutropenias persistentes em pacientes caninos e felinos, principalmente após quimioterapias. Mais trabalhos em relação ao uso deste estimulante em cães e em gatos devem ser desenvolvidos, especialmente em casos ainda controversos, como na hipoplasia de medula óssea decorrente de erliquiose crônica, na FIV e na FeLV. Além disso, deve-se estudar o desenvolvimento do G-CSF recombinante canino e felino, que ainda não estão disponíveis no mercado, que certamente exerceriam contribuições mais significativas para a terapêutica de pequenos animais.

\section{REFERÊNCIAS}

AROCH, I.; HARRUS, S. The use of recombinant human colony stimulating factor and recombinant human erythropoietin in the treatment of severe pancytopenia due to canine monocytic ehrlichiosis. Israel Journal of Veterinary Medicine, v.56, n.2, p.65-69, 2001.

BRAVO, L. et al. Serum granulocyte colony-stimulating factor (G-CSF) and interleukin-1 (IL-1) concentrations after chemotherapy-induced neutropenia in normal and tumorbearing dogs. Experimental Hematology, v.24, n.1, p.11$17,1996$.

BROWN, R.; ROGERS, K.S. Neutropenia in dogs and cats: a retrospective study of 261 cases. Journal of the American Animal Hospital Association, v.37, n.2, p.131-139, 2001.

CHUN, R. et al. Cancer chemotherapy. In: WITHROW, S.J. MACEWEN, E.G. Small animal clinical oncology. 3.ed. Philadelphia: Saunders, 2001. Cap.9, p.92-118.

COUTO, C.G. Management of complications of cancer chemotherapy. Veterinary Clinics of North America Small Animal Practice, v.20, n.4, p.1037-1053, 1990
COUTO, C.G. Complications of cancer chemotherapy. In: NELSON, R.W.; COUTO, C.G. Small animal internal medicine. 3.ed. St.Louis: Mosby, 2003. Cap.80, p.1108-1116.

EICHACKER, P.Q. et al. Cardiopulmonary effects of granulocyte colony-stimulating factor in a canine model of bacterial sepsis. Journal of Applied Physiology, v.77, n.5, p.2366-73, 1994 .

FELDMAN, B.F. et al. Schalm's veterinary hematology. 5.ed. Philadelphia: Lippincott Willians \& Wilkins, 2000. 1344p.

HAMMOND, W.P. et al. A comparison of treatment of canine cyclic hematopoiesis with recombinant human granulocytemacrophage colony-stimulating factor (GM-CSF), G-CSF, interleukin-3, and canine G-CSF. Blood, v.76, n.3, p.523-532, 1990.

HAMMOND, W.P. et al. Chronic neutropenia. A new canine model induced by human granulocyte colony-stimulating factor. Journal of Clinical Investigation, v.87, n.2, p.704-710, 1991.

HARVEY, J.W. Atlas of veterinary hematology - Blood and bone marrow of domestic animals. Philadelphia: Saunders, 2001. 228p.

HELTON, K.A. et al. Griseofulvin toxicity in cats: literature review and report of seven cases. Journal of the American Animal Hospital Association, v.22, n.2, p.453-458, 1986.

HENRY, C.J. et al. Veterinary uses of recombinant human granulocyte colony-stimulating factor. Part I. Oncology. Compendium on Continuing Education for the Practicing Veterinarian, v.20, n.6, p.728-734, 1998.

HOLLAND, M. et al. Pancytopenia associated with administration of captopril to a dog. Journal of the American Veterinary Medical Association, v.208, n.10, p.1683-1686, 1996.

JAIN, N.C. Essentials of veterinary hematology. Philadelphia: Lea \& Febiger, 1993. 417p.

KIM, S.K.; DEMETRI, G.D. Chemotherapy and neutropenia Hematology/Oncology Clinics of North America, v.10, n.2, p.377-395, 1996 .

KUNKLE, G.A.; MEYER, D.J. Toxicity of high doses of griseofulvin in cats. Journal of the American Veterinary Medical Association, v.191, n.3, p.322-323, 1987.

LESTER, S.J.; SEARCY, G.P. Hematologic abnormalities preceding apparent recovery from feline leukemia virus infection. Journal of the American Veterinary Medical Association, v.178, n.5, p.471-474, 1981.

LOTHROP, C.D.JR. et al. Correction of canine cyclic hematopoiesis with recombinant human granulocyte colonystimulating factor. Blood, v.72, n.4, p.1324-1328, 1988.

MAC VITTIE, T.J. et al. Defining the full therapeutic potential of recombinant growth factors in the post radiation-accident environment: the effect of supportive care plus administration of G-CSF. Health Physics, v.89, n.5, p.546-555, 2005. 
MISCHKE, R. et al. Effect of recombinant human granulocyte colony-stimulating factor (rhG-CSF) on leukocyte count and survival rate of dogs with parvoviral enteritis. Research in Veterinary Science, v.70, n.3, p.221-225, 2001 .

MISHU, L. et al. Effects of recombinant canine granulocyte colony-stimulating factor on white blood cell production in clinically normal and neutropenic dogs. Journal of the American Veterinary Medical Association, v.200, n.12, p.1957-1964, 1992.

NOTHDURFT, W. et al. Acceleration of hemopoietic recovery in dogs after extended-field partial-body irradiation by treatment with colony-stimulating factors: rhG-CSF and rhGM-CSF. International Journal of Radiation Oncology, Biology, Physics, v.37, n.5, p.1145-1154, 1997.

OBRADOVICH, J.E. et al. Evaluation of recombinant canine granulocyte colony-stimulating factor as an inducer of granulopoiesis. Journal of Veterinary Internal Medicine, v.5, n.2, p.75-79, 1991.

OBRADOVICH, J.E. et al. Effect of recombinant canine granulocyte colony-stimulating factor on peripheral blood neutrophil counts in normal cats. Journal of Veterinary Internal Medicine, v.7, n.2, p.65-67, 1993.

OGUMA, K. et al. In vitro effects of recombinant human granulocyte colony-stimulating factor on canine neutrophil apoptosis. Veterinary Immunology and Immunopathology, v.108, n.3, p.307-314, 2005.

PEREZ, R.R. et al. A ação do decanoato de nandrolona (Decadurabolin ${ }^{\circledR}$ ) sobre parâmetros hematológicos e proteína total plasmática de ratos (Rattus rattus) com depressão medular induzida após administração de sulfato de vincristina (Oncovin ${ }^{\circledR}$ ). Ciência Rural, v.35, n.3, p.589-595, 2005.

PHILLIPS, K. et al. FIV-infected cats respond to short-term rHuG-CSF treatment which results in anti-G-CSF neutralizing antibody production that inactivates drug activity. Veterinary Immunology and Immunopathology, v.108, n.3, p.357$371,2005$.

QUEZADO, Z. et al. Acute G-CSF therapy is not protective during lethal E. coli sepsis. American Journal of Physiology. Regulatory, Integrative and Comparative Physiology, v.281, n.4, p.1177-1185, 2001.
REWERTS, J.M.; HENRY, C.J. Veterinary uses of recombinant human granulocyte colony-stimulating factor. Part II. Infectious Diseases. Compendium on Continuing Education for the Practicing Veterinarian, v.20, n.7, p.823-827, 1998.

SHELTON, G.H.; LINENBERGER, M.L. Hematologic abnormalities associated with retroviral infections in the cat. Seminars in Veterinary Medicine and Surgery (Small Animal), v.10, n.4, p.220-233, 1995.

SHELTON, G.H. et al. Hematologic manifestations of feline immunodeficiency virus infection. Blood, v.76, n.6, p.1104$1109,1990$.

STOCKHAM, S.L.; SCOTT, M.A. Leukocytes. In: Fundamentals of veterinary clinical pathology. Iowa: Iowa State, 2002. Cap.3, p.49-83.

STORB, R. et al. DLA-identical bone marrow grafts after lowdose total body irradiation: the effect of canine recombinant hematopoietic growth factors. Blood, v.84, n.10, p.35583566, 1994.

SUTTORP, M. et al. Prevention of oestradiol-associated toxicosis in a dalmatian by early intervention with granulocyte colony-stimulating factor. Veterinary Record, v.151, n.8, p.244-245, 2002.

THOMASSON, B. et al. Direct comparison of steady-state marrow, primed marrow, and mobilized peripheral blood for transduction of hematopoietic stem cells in dogs. Human Gene Therapy, v.14, n.17, p.1683-1686, 2003.

WAGNER, J.G.; ROTH, R.A. Neutrophil migration during endotoxemia. Journal of Leukocyte Biology, v.66, n.1, p.10-24, 1999.

WALTON, R.M. et al. Bone marrow cytological findings in four dogs and a cat with hemophagocytic syndrome. Journal of Veterinary Internal Medicine, v.10, n.1, p.7-14, 1996.

WEISS, D.J. Cytologic evaluation of benign and malignant hemophagocytic disorders in canine bone marrow. Veterinary Clinical Pathology, v.30, n.1, p.28-34, 2001.

WEISS, D.J., AIRD, B. Cytologic evaluation of primary and secondary myelodysplastic syndromes in the dog. Veterinary Clinical Pathology, v.30, n.2, p.67-75, 2001. 